\title{
Co-Attentive Multi-Task Learning for Explainable Recommendation
}

\author{
Zhongxia Chen ${ }^{1,2}$, Xiting Wang ${ }^{2 *}$, Xing Xie ${ }^{2}$, Tong Wu ${ }^{3}$, \\ Guoqing $\mathrm{Bu}^{3}$, Yining $\mathrm{Wang}^{3}$ and Enhong Chen ${ }^{1}$ \\ ${ }^{1}$ School of Computer Science and Technology, University of Science and Technology of China, China \\ ${ }^{2}$ Microsoft Research Asia, China \\ ${ }^{3}$ CFETS Information Technology (Shanghai) Co., Ltd., China \\ \{czx87@mail., cheneh@\}ustc.edu.cn, \{xitwan,xing.xie $\} @$ microsoft.com, \\ \{wutong, buguoqing_zh, wangyining_zh\}@ chinamoney.com.cn
}

\begin{abstract}
Despite widespread adoption, recommender systems remain mostly black boxes. Recently, providing explanations about why items are recommended has attracted increasing attention due to its capability to enhance user trust and satisfaction. In this paper, we propose a co-attentive multitask learning model for explainable recommendation. Our model improves both prediction accuracy and explainability of recommendation by fully exploiting the correlations between the recommendation task and the explanation task. In particular, we design an encoder-selector-decoder architecture inspired by human's information-processing model in cognitive psychology. We also propose a hierarchical co-attentive selector to effectively model the cross knowledge transferred for both tasks. Our model not only enhances prediction accuracy of the recommendation task, but also generates linguistic explanations that are fluent, useful, and highly personalized. Experiments on three public datasets demonstrate the effectiveness of our model.
\end{abstract}

\section{Introduction}

Personalized recommendation has become a major technique for helping users handle huge amounts of online content. To improve user experience, it is essential that the recommendation model accurately predicts users' personal preferences for items. Except for accuracy, there is a growing interest in explainability [Alvarez-Melis and Jaakkola, 2018]. Evidence has shown that explanations about why the items are recommended can increase user trust, improve satisfaction, and persuade the users to buy or try an item [Rago et al., 2018].

The recent attention on explainability has lead to the development of a series of explainable recommendation models. A fundamental question explainable recommendation aims to answer is how we balance accuracy and explainability. Most existing methods consider the two goals in separate steps or only focus on one of the goals, which limits their effectiveness. In particular, let us consider the two most widely used frameworks: post-hoc and embedded.

\footnotetext{
${ }^{*}$ Xiting Wang is the corresponding author
}

Item: Last Stand of the $300 \quad$ User interest: war, history, documentary
$\begin{array}{ll}\text { (a) Post-hoc } & \text { Alice and } 7 \text { of your friends like this. } \\ \text { Because you watched Spartacus, we recommend Last Stand of the } 300 .\end{array}$
$\begin{array}{ll}\text { (b) Embedded-F } & \text { You might be interested in documentary, on which this item performs well. } \\ \text { (c) Embedded-S } & \text { I agree with several others that this is a good companion to the movie. } \\ \text { (d) Joint } & \text { This is a very good movie. } \\ \text { (e) Ours } & \text { This is a very good documentary about the battle of thermopylae. } \\ - \text { Pre-defined template } \quad \text { Retrieved from explanations written by others Generated by RNNs }\end{array}$

Figure 1: Explanations provided by different methods: (a) posthoc methods [Sharma and Cosley, 2013; Peake and Wang, 2018]; (b) a feature-level embedded method [Zhang et al., 2014]; (c) a sentence/review-level embedded method [Chen et al., 2018]; (d) a joint model [Li et al., 2017]; and (e) our model. Underlined words are related with user interest. Our model generates informative and personalized explanations that are relevant to the item.

Post-hoc methods explain a black-box model after it is trained [Vig et al., 2009; Sharma and Cosley, 2013; Peake and Wang, 2018]. They consider accuracy and explainability in separate steps and provide explanations based on the model outputs. Typically, the rich information embedded inside the recommendation models are ignored and the explanations are selected from a set of pre-defined templates. As shown in Fig. 1(a), the templates are often readable and persuasive. However, the explanations may not reflect the model's actual reasoning and the diversity of the explanations are limited.

Embedded methods integrate the explanation process into the construction of the recommendation model [McAuley and Leskovec, 2013; Zhang et al., 2014; He et al., 2015; Chen et al., 2018; Wang et al., 2018a]. These methods focus on recommendation accuracy. Their explanations consist of features or sentences that are important for improving accuracy (Fig. 1(b)(c)). Since explainability is not included in the optimization goal, it is difficult to guarantee the quality of the explanations, e.g., consistency [Wang et al., 2018b]. Moreover, the embedded methods are retrieval-based (i.e., selecting from original dataset). They may fail to provide a highly personalized explanation when data is sparse and may suffer from legal issues (e.g., copyright) in certain scenarios.

The purpose of this paper is to illustrate how to effectively optimize accuracy and explainability in a joint and unified framework. The key idea is that fully exploiting the correlations between the recommendation task and the explanation task potentially enables both tasks to be better off than when they are considered separately. While the idea seems 
promising, achieving this objective is challenging. A simple multi-task learning framework that only shares latent representations such as user and item embeddings between the two tasks cannot achieve desirable results. As shown in Fig. 1(d), the generated explanations are usually quite general, i.e., they do not contain specific information about key features of the item. This is because 1) the shared representations are not explainable and fail to provide explicit constraints on the explanations and 2) user and item embeddings do not contain sufficient information about deep user-item interactions.

To address the aforementioned issues, we propose a $\mathbf{C o}$

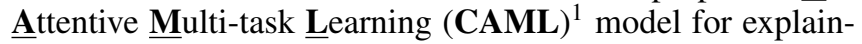
able recommendation, which enhances both accuracy and explainability of explainable recommendation by tightly coupling the recommendation task and the explanation task.

The major contributions of this paper are:

First, we design an encoder-selector-decoder architecture for multi-task learning. Our architecture design is inspired by models in cognitive psychology. Cognitive scientists have shown that a human's cognitive process consists of three major sub-processes [Lang, 2000]. The three sub-processes correspond to our encoder, selector, and decoder. In explainable recommendation, the decoder is responsible for deciding the predicted rating (recommendation task) and generating the explanations (explanation task). The selector serves as the transferred cross knowledge for both tasks. The architecture tightly couples the two tasks in a natural way.

Second, we propose a hierarchical co-attentive selector to effectively control the cross knowledge transfer for both tasks. The selector models deep level interactions between the users and items. In particular, it identifies reviews and concepts (cross knowledge) that are important for the useritem pair based on co-attention. Compared with traditional methods such as REINFORCE [Williams, 1992], our model does not suffer from slow convergence and high variance because we use hierarchical multi-pointer networks [Vinyals $e t$ al., 2015] for review and concept selection.

Finally, extensive experiments demonstrate that our method improves both explainability and accuracy. As shown in Fig. 1(e), our method is able to generate fluent explanations that are informative, highly personalized, and relevant with the item. Numerical experiments demonstrate that the explanations of CAML increases BLEU scores by $14.6 \%$ to $35.9 \%$. Human evaluation shows that our explanations are more fluent and much more useful compared with the state-of-art generative model (explainability). Moreover, CAML consistently improves recommendation accuracy on three public datasets compared with 7 baselines (accuracy).

In the rest of the paper, we first introduce the problem definition and our model. We then describe the experiments and conclusion. The related work section is omitted because most existing works have been discussed in the introduction.

\section{Problem Formulation}

We formulate our problem as follows.

Input. The input of our model consists of the user set $U$, the item set $V$, the reviews, and the concepts in the reviews:

\footnotetext{
${ }^{1}$ Source code: https://github.com/3878anonymous/CAML.
}

- Each user is represented by its ID $u \in U$ and each item is denoted by the item ID $v \in V$.

- The reviews of a user $u$ are $\left(\mathcal{D}_{u, 1}, \ldots, \mathcal{D}_{u, l_{d}}\right)$, where $l_{d}$ denotes the maximum number of reviews. Each review $\mathcal{D}_{u, i}$ is denoted by a set of words in the review. Similarly, $\left(\mathcal{D}_{v, 1}, \ldots, \mathcal{D}_{v, l_{d}}\right)$ represents the reviews of item $v$.

- The concepts are a subset of words that correspond to important explicit features mentioned in the review. For example, the review "This is a great little comedy with a catchy song" contains two concepts: comedy and song. We derive the concepts by utilizing Microsoft Concept $\mathrm{Graph}^{2}$ [Wu et al., 2012; Wang et al., 2015], a widely used knowledge graph with about 18 million concepts or instances. We map the n-grams in the reviews to concepts or instances in the concept graph and filter concepts that are rarely used or not informative.

Output. Given a user-item pair $(u, v)$, our model predicts:

- The rating $r$ that reflects how much $u$ likes $v$.

- The linguistic explanation $Y=\left(y_{1}, y_{2}, \ldots, y_{T}\right)$ that illustrates why user $u$ likes or dislikes item $v$. Here $y_{k}$ denotes the $k$-th word in the explanation. During training, ground truth explanations can be set to reviews the users write for the corresponding item [Li et al., 2017].

\section{Model Description}

In this section, we introduce CAML, a Co-Attentive Multitask Learning model for explainable recommendation. Our model improves both prediction accuracy and explainability by fully exploiting the correlations between the recommendation task and explanation task. To achieve these goals, we design an encoder-selector-decoder architecture. As shown in Fig. 2, our model consists of three components, each corresponds to one major sub-process of the informationprocessing model in cognitive psychology [Lang, 2000].

- In the encoder, we embed the words, reviews, and the implicit factors of users and items. The encoder corresponds to the sub-process of encoding in information processing.

- The multi-pointer co-attention selector identifies reviews and concepts that are important for both users and items by leveraging hierarchical multi-pointer co-attention. The identified concepts serve as the cross knowledge for the two tasks. This component corresponds to the storage sub-process in information processing, which distinguishes important pieces of encoded information and stores them properly through associations and links (i.e., networks).

- The multi-task decoder is responsible for predicting the ratings and generating the explanations based on the key concepts extracted by the selector. In information processing, this corresponds to the retrieval sub-process, which reactivates important information for decision making.

Next, we will introduce each component and how we jointly optimize them in an end-to-end framework.

\subsection{Encoder}

Word encoder. We use a look up layer to transform a word ID into a word embedding $\boldsymbol{\omega} \in \mathcal{R}^{l_{w}}$. Here, $l_{w}$ is the dimensionality of word representations.

\footnotetext{
${ }^{2}$ https://concept.research.microsoft.com/
} 


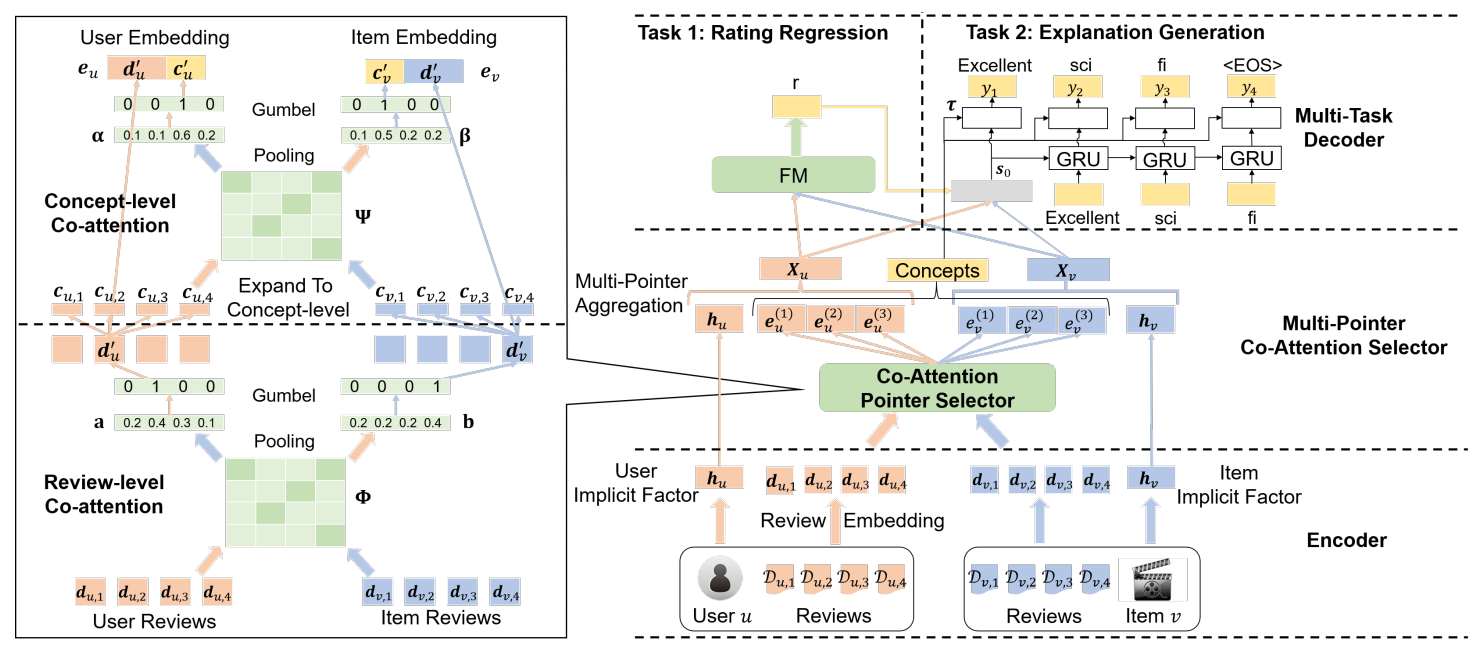

Figure 2: Framework of the proposed model for explainable recommendation.

Review encoder. We calculate an initial review embedding by following [Tay et al., 2018]. Given review $\mathcal{D}_{u, i}$, the corresponding review embedding $\mathbf{d}_{u, i}$ can be calculated by $\mathbf{d}_{u, i}=\sum_{\boldsymbol{\omega} \in \mathcal{D}_{u, i}} \boldsymbol{\omega}$. Summing up the embeddings of related words to derive an initial review embedding balances both effectiveness and efficiency.

User/Item implicit factor encoder. Explicit factors such as reviews may not contain all information about a user or an item. To complement explicit factors, we introduce implicit user or item factors by following [Zhang et al., 2014]. Specifically, we use a look up layer to transform user $u$ (or item $v$ ) into implicit representation $\mathbf{h}_{u} \in \mathcal{R}^{l_{u}}\left(\right.$ or $\mathbf{h}_{v} \in \mathcal{R}^{l_{v}}$ ).

\subsection{Multi-Pointer Co-Attention Selector}

We model the cross knowledge transferred for the two tasks by identifying (selecting) reviews and concepts that are important for user-item pairs. Our method is based on the multipointer co-attention networks [Tay et al., 2018], which 1) are effective in modeling a deeper level of pairwise interactions and 2) have better convergence properties compared with alternatives such as REINFORCE [Williams, 1992]. We first show how we extend the networks to hierarchically select reviews and then concepts. Next, we discuss how we support selection and aggregation of multiple reviews and concepts.

\section{Review-level Co-attention Pointer}

Given user review embeddings $\mathbf{d}_{u, 1}, \ldots, \mathbf{d}_{u, l_{d}}$ and item review embeddings $\mathbf{d}_{v, 1}, \ldots, \mathbf{d}_{v, l_{d}}$, we first calculate co-attention weight matrix $\boldsymbol{\Phi} \in \mathcal{R}^{l_{d} \times l_{d}}$ among user-item review pairs. Specifically, the $(i, j)$-th entry of $\boldsymbol{\Phi}$ is computed by:

$$
\phi_{i, j}=F\left(\mathbf{d}_{u, i}\right)^{\mathrm{T}} \mathbf{W}_{d} F\left(\mathbf{d}_{v, j}\right)
$$

Here, $\mathbf{W}_{d} \in \mathcal{R}^{l_{w} \times l_{w}}$ is the weight matrix and $F$ is a feedforward neural network with $l_{F}$ layers.

Max pooling over the co-attention matrix selects reviews with the maximum correlations with all reviews:

$$
a_{i}=\max _{j=1, \ldots, l_{d}} \phi_{i, j} \quad \text { and } \quad b_{j}=\max _{i=1, \ldots, l_{d}} \phi_{i, j}
$$

To turn the unnormalized vectors $\mathbf{a}=\left(a_{1}, \ldots, a_{l_{d}}\right)$ and $\mathbf{b}=\left(b_{1}, \ldots, b_{l_{d}}\right)$ into a probability distribution, the most commonly used function is softmax. However, selecting reviews (i.e., applying the arg max function) from the softmax distribution makes the model non-differentiable. To address this issue, the Straight-Through Gumbel-Softmax function [Jang et al., 2017] is used for attention learning:

$$
q_{i}=\frac{\exp \left(\frac{a_{i}+g_{i}}{\tau}\right)}{\sum_{j=1}^{n_{m}} \exp \left(\frac{a_{j}+g_{i}}{\tau}\right)}
$$

where $g_{i}$ is the Gumbel noise, $\tau$ is the temperature parameter which controls the smoothness of the vector $\mathbf{q}$. If $\tau$ is close to zero, $\mathbf{q}$ becomes close to a one-hot vector. In the forward pass, $\arg \max$ is leveraged to gain a pointer $\mathbf{z}$ from $\mathbf{q}$ :

$$
z_{i}= \begin{cases}1, & i=\arg \max _{j}\left(q_{j}\right) \\ 0, & \text { otherwise }\end{cases}
$$

We denote this function as $\operatorname{Gumbel}(\mathbf{a})=\mathbf{z}$.

In the backward pass, the function approximates the gradients of $\mathbf{z}$ by using the gradients of $\mathbf{q}$ to ensure end-toend model training [Jang et al., 2017]. The review-level coattentional user embedding $\mathbf{d}_{u}^{\prime}$ and item embedding $\mathbf{d}_{v}^{\prime}$ are:

$$
\mathbf{d}_{u}^{\prime}=(\operatorname{Gumbel}(\mathbf{a}))^{\mathrm{T}} \mathbf{D}_{u} \text { and } \mathbf{d}_{v}^{\prime}=(\operatorname{Gumbel}(\mathbf{b}))^{\mathrm{T}} \mathbf{D}_{v}
$$

Here, the $i$-th row of $\mathbf{D}_{u} \in \mathcal{R}^{l_{d} \times l_{w}}$ (or $\mathbf{D}_{v}$ ) is $\mathbf{d}_{u, i}^{\mathrm{T}}\left(\right.$ or $\mathbf{d}_{v, i}^{\mathrm{T}}$ ).

\section{Concept-level Co-attention Pointer}

Suppose that the selected user review contains concepts $\mathbf{c}_{u, 1}, \ldots, \mathbf{c}_{u, l_{c}}$. Here $\mathbf{c}_{u, i}$ denotes the word embedding of the $i$-th selected concept. The concepts of the selected item review are $\mathbf{c}_{v, 1}, \ldots, \mathbf{c}_{v, l_{c}}$. The concept co-attention matrix $\boldsymbol{\Psi} \in \mathcal{R}^{l_{c} \times l_{c}}$ is calculated by

$$
\psi_{i, j}=F\left(\mathbf{c}_{u, i}\right)^{\mathrm{T}} \mathbf{W}_{c} F\left(\mathbf{c}_{v, j}\right)
$$

where $\mathbf{W}_{c} \in \mathcal{R}^{l_{w} \times l_{w}}$ is the weight matrix. Next, we calculate the weights of the concepts by aggregating $\Psi$. We investigate different pooling strategies and find mean pooling to perform the best. Thus, mean pooling is used to calculate the weights of the concepts $\boldsymbol{\alpha}$ and $\boldsymbol{\beta}$ :

$$
\alpha_{i}=\frac{1}{l_{c}} \sum_{j=1, \ldots, l_{c}} \psi_{i, j} \quad \text { and } \quad \beta_{j}=\frac{1}{l_{c}} \sum_{i=1, \ldots, l_{c}} \psi_{i, j}
$$


Here, $\alpha_{i}$ is the $i$-th entry of $\boldsymbol{\alpha}$ and $\beta_{j}$ is the $j$-th entry of $\boldsymbol{\beta}$. The concept-level co-attentional embeddings are:

$$
\mathbf{c}_{u}^{\prime}=(\operatorname{Gumbel}(\boldsymbol{\alpha}))^{\mathrm{T}} \mathbf{C}_{u} \text { and } \mathbf{c}_{v}^{\prime}=(\operatorname{Gumbel}(\boldsymbol{\beta}))^{\mathrm{T}} \mathbf{C}_{v}
$$

where the $i$-th row of $\mathbf{C}_{u} \in \mathcal{R}^{l_{c} \times l_{w}}\left(\right.$ or $\left.\mathbf{C}_{v}\right)$ is $\mathbf{c}_{u, i}^{\mathrm{T}}\left(\right.$ or $\mathbf{c}_{v, i}^{\mathrm{T}}$ ).

\section{Multi-pointer Aggregation}

Since a user may consider multiple reviews and concepts when s/he provides ratings or reviews, we need to support selection and aggregation of multiple pointers. Consider the co-attentional user embedding $\mathbf{e}_{u}=\left[\mathbf{d}_{u}^{\prime} ; \mathbf{c}_{u}^{\prime}\right]$ that combines review-level and concept-level user embedding. In the multipointer setting, we run our co-attention pointer selector multiple times with different Gumbel noises to get multiple samples: $\left\{\mathbf{e}_{u}^{(1)}, \ldots, \mathbf{e}_{u}^{\left(n_{p}\right)}\right\}$. Similarly, we obtain a set of samples for the co-attentional item embedding: $\left\{\mathbf{e}_{v}^{(1)}, \ldots, \mathbf{e}_{v}^{\left(n_{p}\right)}\right\}$. We then use a non-linear layer to aggregate multiple samples:

$$
\begin{aligned}
\overline{\mathbf{e}}_{u} & =\sigma\left(\mathbf{W}_{p}\left[\mathbf{e}_{u}^{(1)}, \ldots, \mathbf{e}_{u}^{\left(n_{p}\right)}\right]+\mathbf{b}_{p}\right) \\
\overline{\mathbf{e}}_{v} & =\sigma\left(\mathbf{W}_{p}\left[\mathbf{e}_{v}^{(1)}, \ldots, \mathbf{e}_{v}^{\left(n_{p}\right)}\right]+\mathbf{b}_{p}\right)
\end{aligned}
$$

where $\mathbf{W}_{p} \in \mathcal{R}^{l_{p} \times 2 n_{p} l_{w}}$ and $\mathbf{b}_{p} \in \mathcal{R}^{l_{p}}$. The aggregated coattentional user and item embeddings are used in both rating prediction and explanation generation.

\subsection{Multi-Task Decoder}

We propose a multi-task decoder that predicts ratings and generates explanations based on representations learned in the encoder and selector. We consider two types of user and item embeddings: the co-attentional embeddings $\overline{\mathbf{e}}_{u}$ and $\overline{\mathbf{e}}_{v}$ which are learned from explicit factors (reviews), and implicit factor representations $\mathbf{h}_{u}$ and $\mathbf{h}_{v}$. To simultaneously capture an item's explicit and implicit factors, the user and item embeddings are expressed as $\mathbf{x}_{u}=\left[\overline{\mathbf{e}}_{u} ; \mathbf{h}_{u}\right]$ and $\mathbf{x}_{v}=\left[\overline{\mathbf{e}}_{v} ; \mathbf{h}_{v}\right]$.

\section{Rating Prediction}

Factorization machine (FM) [Rendle, 2010] is used as the rating prediction method to model pairwise feature interactions:

$$
r=f(\mathbf{x})=w_{0}+\sum_{i=1}^{n} w_{i} x_{i}+\sum_{i=1}^{n} \sum_{j=i+1}^{n}\left\langle\mathbf{m}_{i}, \mathbf{m}_{j}\right\rangle x_{i} x_{j}
$$

Here, $x_{i} \in \mathcal{R}$ is the $i$-th entry of $\mathbf{x}=\left[\mathbf{x}_{u} ; \mathbf{x}_{v}\right], w_{0} \in \mathcal{R}$ is the bias, $w_{i} \in \mathcal{R}$ and $\mathbf{m}_{i} \in \mathcal{R}^{k}$ are parameters to be learned. The loss function of our rating prediction task is formulated as:

$$
\mathcal{L}_{r}=\frac{1}{2|\Omega|} \sum_{(u, v) \in \Omega}\left(r-r_{*}\right)^{2}
$$

where $\Omega$ represents the training set and $r_{*}$ is the true rating.

\section{Explanation Generation}

We generate explanation $Y$ based on user embedding $\mathbf{x}_{u}$, item embedding $\mathbf{x}_{v}$, predicted rating $r$, and concepts chosen in the selector. We first show how we use Gated Recurrent Unit (GRU) [Chung et al., 2014] to obtain word sequences. Then, we introduce two related losses used in the training process.

$G R U$. GRU demonstrates high capability in text generation tasks. To incorporate $\mathbf{x}_{u}, \mathbf{x}_{v}$, and $r$ into GRU, we compute the initial hidden state $\mathbf{s}_{0}$ by:

$$
\mathbf{s}_{0}=\tanh \left(\mathbf{W}_{u} \mathbf{x}_{u}+\mathbf{W}_{v} \mathbf{x}_{v}+\mathbf{W}_{r} \hat{\mathbf{r}}+\mathbf{b}_{s}\right)
$$

Here, $\hat{\mathbf{r}}$ is a vector representation of $r . \quad \hat{\mathbf{r}}$ is calculated by rounding $r$ into an integer (e.g., $3.14 \rightarrow 3$ ) and converting it to a one-hot vector. $\mathbf{W}_{u}, \mathbf{W}_{v}, \mathbf{W}_{r}$, and $\mathbf{b}_{s}$ are parameters to be learned. The hidden state $\mathbf{s}_{t}$ at time $t$ is calculated recursively:

$$
\mathbf{s}_{t}=\operatorname{GRU}\left(\mathbf{s}_{t-1}, \boldsymbol{\omega}_{y_{t}}\right)
$$

where $\boldsymbol{\omega}_{y_{t}}$ is the embedding of the word $y_{t}$ generated at time $t$. This hidden state is fed into the output layer to generate the output word distribution $\mathbf{o}_{t}$ as time $t$ :

$$
\mathbf{o}_{t}=\zeta\left(\mathbf{W}_{o} \mathbf{s}_{t-1}+\mathbf{b}_{o}\right)
$$

where $\mathbf{W}_{o} \in \mathcal{R}^{|\mathcal{V}| \times l_{t}}, \mathbf{b}_{o} \in \mathcal{R}^{|\mathcal{V}|}, \zeta$ is the softmax function and $|\mathcal{V}|$ is the vocabulary size. During testing, beam search is used to find the best explanation $Y=\left(y_{1}, \ldots, y_{T}\right)$ with the maximum log-likelihood $\sum_{t=1}^{T} \log o_{t, y_{t}}$.

Concept relevance loss $\mathcal{L}_{c}$. During training, $\mathcal{L}_{c}$ is used to increase the probability that the selected concepts appear in $Y$. Let us denote the selected concept vector as $\tau \in \mathcal{R}^{|\mathcal{V}|} . \tau_{k}$ is 1 if the $k$-th word is a concept and has been selected by at least one pointer and is 0 otherwise. $\mathcal{L}_{c}$ is computed by:

$$
\mathcal{L}_{c}=\frac{1}{|\Omega|} \sum_{(u, v) \in \Omega} \sum_{t=1}^{T}\left(\max _{k}\left(-\tau_{k} \log o_{t, k}\right)\right)
$$

Negative log-likelihood loss $\mathcal{L}_{n} . \mathcal{L}_{n}$ is widely used to ensure that the generated words are similar to the ground truth ones. Let $y_{t *}$ denote the ground truth word at time $t$, we have

$$
\mathcal{L}_{n}=\frac{1}{|\Omega|} \sum_{(u, v) \in \Omega} \sum_{t=1}^{T}\left(-\log o_{t, y_{t *}}\right)
$$

\subsection{Joint Learning}

Different types of loss functions are linearly combined to jointly learn two tasks in an end-to-end manner:

$$
\mathcal{L}=\mathcal{L}_{r}+\lambda_{c} \mathcal{L}_{c}+\lambda_{n} \mathcal{L}_{n}+\lambda_{l}\|\Theta\|_{2}^{2}
$$

where $\lambda_{c}, \lambda_{n}$, and $\lambda_{l}$ are weights that balance the importance of different losses. $\Theta$ contains all model parameters. We choose Adam [Kingma and $\mathrm{Ba}, 2015$ ] as the training optimizer because it empirically outperforms other stochastic optimization methods.

\section{Experiments}

\subsection{Experimental Settings}

\section{Datasets}

Three publicly available datasets from different domains are used in our evaluation:

- Electronics is the part of Amazon dataset ${ }^{3}$ that focuses on electronic products. We use the 5-core version where users and items have no fewer than 5 reviews.

\begin{tabular}{cccc}
\hline & Electronics & Movies\&TV & Yelp-2016 \\
\hline Users & 192,403 & 123,960 & 677,379 \\
Items & 63,001 & 50,052 & 84,693 \\
Reviews & $1,688,117$ & $1,697,533$ & $2,530,843$ \\
Concepts & 652 & 791 & 1,004 \\
\hline
\end{tabular}

Table 1: Statistics of three public datasets.

\footnotetext{
${ }^{3}$ http://jmcauley.ucsd.edu/data/amazon/
} 
Proceedings of the Twenty-Eighth International Joint Conference on Artificial Intelligence (IJCAI-19)

\begin{tabular}{|c|c|c|c|c|c|c|c|c|c|c|}
\hline \multirow{2}{*}{ Datasets } & \multirow{2}{*}{ Criteria } & \multicolumn{3}{|c|}{ Retrieval } & \multirow{2}{*}{$\begin{array}{c}\text { Generative } \\
\text { NRT }\end{array}$} & \multicolumn{3}{|c|}{ Ours } & \multicolumn{2}{|c|}{ Improvement (\%) } \\
\hline & & LexRank & NARRE & RLRec & & CAML-G & CAML-C & CAML & $\Delta_{\text {Retrieval }}$ & $\Delta_{\text {Generative }}$ \\
\hline \multirow{5}{*}{ Electronics } & BLEU & 1.44 & 1.45 & 1.45 & 1.33 & 1.79 & 1.92 & 1.97 & +35.9 & +48.1 \\
\hline & ROUGE-1 & 14.22 & 15.19 & 11.12 & 17.39 & 18.64 & 19.00 & 19.26 & +26.8 & +10.8 \\
\hline & ROUGE-2 & 3.60 & 3.29 & 1.60 & 3.50 & 3.63 & 3.78 & 3.81 & +5.8 & +8.9 \\
\hline & ROUGE-L & 13.70 & 13.28 & 9.70 & 15.71 & 16.37 & 16.63 & 16.75 & +22.3 & +6.6 \\
\hline & ROUGE-SU4 & 4.38 & 5.25 & 3.13 & 5.97 & 6.26 & 6.43 & 6.47 & +23.2 & +8.4 \\
\hline \multirow{5}{*}{ Movies\&TV } & BLEU & 1.78 & 1.75 & 1.73 & 1.60 & 1.94 & 2.04 & 2.04 & +14.6 & +27.5 \\
\hline & ROUGE-1 & 15.68 & 15.31 & 11.61 & 18.09 & 18.86 & 19.14 & 19.32 & +23.2 & +6.8 \\
\hline & ROUGE-2 & 2.45 & 3.62 & 3.84 & 4.30 & 4.48 & 4.43 & 4.58 & +19.3 & +6.5 \\
\hline & ROUGE-L & 12.46 & 12.99 & 10.06 & 16.00 & 16.41 & 16.48 & 16.69 & +28.5 & +4.3 \\
\hline & ROUGE-SU4 & 5.24 & 5.79 & 4.98 & 6.29 & 6.52 & 6.57 & 6.71 & +15.9 & +6.7 \\
\hline \multirow{5}{*}{ Yelp } & BLEU & 0.97 & 1.13 & 1.13 & 1.31 & 1.50 & 1.57 & 1.58 & +39.8 & +20.6 \\
\hline & ROUGE-1 & 11.06 & 10.55 & 9.28 & 13.31 & 13.93 & 14.15 & 14.24 & +28.8 & +7.0 \\
\hline & ROUGE-2 & 2.42 & 2.66 & 1.93 & 3.05 & 3.42 & 3.47 & 3.50 & +31.6 & +14.8 \\
\hline & ROUGE-L & 10.15 & 9.30 & 8.18 & 12.13 & 12.70 & 12.84 & 12.90 & +27.1 & +6.3 \\
\hline & ROUGE-SU4 & 3.58 & 3.85 & 3.10 & 4.60 & 4.92 & 5.00 & 5.03 & +30.6 & +9.3 \\
\hline
\end{tabular}

Table 2: Evaluation results for explanation generation. CAML-G and CAML-C are two variants of our method. $\Delta_{\text {Retrieval }}$ and $\Delta_{\text {Generative }}$ denote the relative improvement of CAML over the most competitive retrieval baseline and the generative baseline.

- Movies\&TV is also from the Amazon 5-core dataset. This dataset focuses on movies and TVs.

- Yelp is a larger and much sparser dataset which contains restaurant reviews from Yelp Challenge $2016^{4}$.

For each dataset, we keep the top 40,000 most frequent words. We filter both rare concepts (occurring in less than $0.5 \%$ of reviews) and domain-dependent frequent concepts (e.g., movie and film in dataset Movies\&TV). The statistics of the datasets are shown in Table 1.

\section{Baselines}

To evaluate explainability, we compare CAML with both retrieval methods and a generative method.

- Retrieval. Retrieval methods select linguistic explanations from review comments in the training set. Three retrieval methods are considered. The first is Lexrank [Erkan and Radev, 2004], a stochastic graph-based method for computing relative importance of textual units. The other baselines are NARRE [Chen et al., 2018] and RLRec [Wang et al., 2018b], which obtain the important sentences or reviews by employing the attention mechanism.

- Generative. Generative methods create explanations by using RNNs. The state-of-art generative method is NRT [Li et al., 2017], which generates an explanation based on rating and the word-level distribution of the review.

To evaluate the accuracy of rating prediction, we compare CAML with two groups of baselines:

- $C F$. The first group consists of Collaborative Filtering (CF) methods. These methods require only the observed ratings for prediction. In particular, we consider three popular CF methods: PMF [Mnih and Salakhutdinov, 2008], NMF [Lee and Seung, 2001], and SVD++ [Koren, 2008].

- Neural. The second group contains neural models that leverage review comments for rating prediction. Four state-of-the-art methods are considered: MPCN [Tay et al., 2018], NARRE, RLRec, and NRT.

\section{Evaluation Criteria}

To measure explainability, we leverage $B L E U$ [Papineni et $a l ., 2002$ ] and ROUGE [Lin, 2004], which are widely used in

\footnotetext{
${ }^{4}$ https://www.yelp.com/dataset/challenge
}

\begin{tabular}{|c|c|}
\hline \multicolumn{2}{|c|}{ Case 1. User interest: horror, night, fun } \\
\hline NRT & I'll admit it. \\
\hline CAML & $\begin{array}{l}\text { I am a huge fan of horror movies, and this is one } \\
\text { of my favorite movies. }\end{array}$ \\
\hline Truth & Remember when horror movies were fun? \\
\hline \multicolumn{2}{|c|}{ Case 2. User interest: humor, scenery, main character } \\
\hline NRT & I love this series. \\
\hline CAML & If you like british humor, you will love this series. \\
\hline Truth & This is very british humor. \\
\hline \multicolumn{2}{|c|}{ Case 3. User interest: story line, cartoon, worth } \\
\hline NRT & I enjoyed this series as much as the first one. \\
\hline CAML & $\begin{array}{l}\text { I enjoyed this movie, the animation was great and } \\
\text { the story line was very good. }\end{array}$ \\
\hline Truth & Great price and the animation was cool. \\
\hline
\end{tabular}

Table 3: Explanations generated by NRT and CAML. "Truth" represents the ground truth explanation. Bolded words are concepts.

machine translation and natural language generation to evaluate the similarity between ground truth texts and generated texts [Li et al., 2016; Li et al., 2017]. Following [Li et al., 2017], we set the ground truth explanations as the first sentences in the reviews. Li et al. also consider tips (short comments provided by users) as ground truth explanations. We ignore tips because most of them are not informative (e.g., "This is a good movie"). Note that the ground truth reviews $\left(\mathcal{D}_{u, v}\right)$ will be excluded from the inputs $\left(\mathcal{D}_{u}\right.$ and $\left.\mathcal{D}_{v}\right)$ to prevent leak of information. We use F-measures of ROUGE1, ROUGE-2, ROUGE-L and ROUGE-SU4 to evaluate the explanations in different granularities. Larger BLEU and ROUGE scores indicate better explainability.

The accuracy of rating prediction is evaluated by employing Root Mean Square Error (RMSE) [Chen et al., 2018], which measures the deviation between the predicted ratings $r$ and the ground truth ratings $r_{*}$.

\section{Implementation Details}

We implement CAML by using Tensorflow ${ }^{5}$. The models are trained on NVIDIA Tesla P100. We randomly choose $80 \%$ of samples as the training data, $10 \%$ as validation and $10 \%$ for testing on each dataset. We initialize the hyper-parameters for the baselines by following the corresponding paper and

\footnotetext{
${ }^{5}$ https://www.tensorflow.org
} 
Proceedings of the Twenty-Eighth International Joint Conference on Artificial Intelligence (IJCAI-19)

\begin{tabular}{|c|c|c|c|c|c|c|c|c|c|c|c|c|}
\hline \multirow{2}{*}{ Datasets } & \multicolumn{3}{|c|}{$\mathbf{C F}$} & \multicolumn{4}{|c|}{ Neural Models } & \multicolumn{3}{|c|}{ Ours } & \multicolumn{2}{|c|}{ Improvement (\%) } \\
\hline & PMF & NMF & SVD++ & MPCN & NARRE & RLRec & NRT & CAML-R & CAML-C & CAML & $\Delta_{C F}$ & $\Delta_{\text {Neural }}$ \\
\hline Electronics & 2.065 & 1.170 & 1.105 & 1.105 & 1.103 & 1.102 & 1.091 & 1.085 & 1.086 & 1.085 & +1.8 & +0.6 \\
\hline Movies\&TV & 1.250 & 1.089 & 1.013 & 1.001 & 0.999 & 1.012 & 0.990 & 0.990 & 0.987 & 0.987 & +2.6 & +0.3 \\
\hline Yelp & 1.829 & 1.290 & 1.193 & 1.193 & 1.190 & 1.220 & 1.186 & 1.180 & 1.173 & 1.173 & +1.7 & +1.1 \\
\hline
\end{tabular}

Table 4: Numerical results for rating prediction (RMSE). CAML-R and CAML-C are two variants of our model. $\Delta_{C F}$ and $\Delta_{N e u r a l}$ represent the relative improvement of CAML over the most competitive CF baseline and neural baseline.

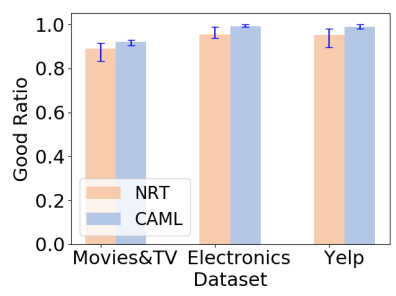

(a) Fluency

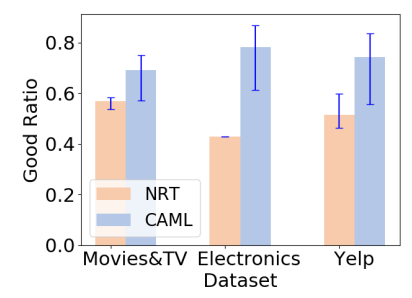

(b) Usefulness
Figure 3: Human evaluation results on explanation quality.

carefully tune them to ensure that they achieve the optimal performance. The initial learning rate is set to $10^{-3}$. The number of pointers is tested in $[1,2,3,4,5]$ for all datasets. For model regularization, the dropout rate is set to 0.2 and the L2 regularization is a fixed number $10^{-6}$. We set the rating prediction and the explanation generation as the same weight 1.0 , and tune $\lambda_{c}$ among [0.01, 0.05, 0.1, 0.2, 0.5].

A more comprehensive description of experimental settings can be found in the supplementary material ${ }^{6}$.

\subsection{Explainability Study}

Overall performance. Table 2 shows that CAML consistently outperforms baselines in terms of BLEU and ROUGE scores on different datasets. Take BLEU as an example, CAML achieves $14.6 \%$ to $39.8 \%$ improvement over the most competitive retrieval method and obtains $20.6 \%$ to $48.1 \%$ improvement over the state-of-art generative method. This illustrates the effectiveness of our encoder-selector-decoder architecture and the multi-pointer co-attention selector. Our method consistently outperforms NRT because it learns deep user-item interactions and provides explicit constraints on the explanations to improve the explanation quality.

Human evaluation w.r.t. fluency and usefulness. With the help of a vendor company, we hire three experienced assessors. We sample 100 test cases from the three datasets and ask the assessors to evaluate if the generated sentences are fluent and useful for helping them decide whether they will try the item or not. The assessors do not know each other and are not aware of which explanation comes from which method (the order of the explanations are randomly shuffled). Fig. 3 shows our model outperforms the state-of-art generative method NRT in terms of both fluency and usefulness on all three datasets. Here, good ratio denotes the percentage of sentences that are considered fluent or useful. The Kappa scores for fluency and usefulness are 0.514 and 0.465 , respectively, showing moderate agreements among assessors.

Case analysis. Table 3 shows examples of explanations generated by NRT and our method (Movies\&TV dataset). For

\footnotetext{
${ }^{6}$ The supplemental material is available at https://github.com/ 3878anonymous/CAML/blob/master/supplemental.pdf
}

each user in the three cases, we display three concepts that $\mathrm{s} /$ he most frequently mentioned in the reviews (training set) to reveal her/his interest. This table shows that our model can select the concepts that fit the user interests, and generate explanations that contain one or multiple appropriate concepts. Compared with NRT, our explanations are informative, useful and similar with the ground truth explanations.

\subsection{Accuracy of Rating Prediction}

The evaluation results of recommendation accuracy is shown in Table 4. In general, neural models outperform traditional CF methods. This is because that the neural models learn more effective representations of users and items through deeper architectures. Moreover, all neural models leverage additional information (i.e., reviews).

Our model consistently achieves the highest accuracy on all three datasets and outperforms NRT by $0.9 \%$ on average. This demonstrates the effectiveness of our encoder-selectordecoder architecture and our approach for modeling explicit user-item interactions through hierarchical co-attention.

\subsection{Ablation Analysis}

Effectiveness of multi-task learning. We compare our multi-task learning model with two variants that consider only one task. The first variant, CAML-G considers only explanation generation and removes the rating prediction loss $\mathcal{L}_{r}$ from Eq. (18). The second variant, CAML-R takes into account only rating prediction and removes explanation generation losses $\mathcal{L}_{c}$ and $\mathcal{L}_{n}$ from Eq. (18). Tables 2 and 4 show that our multi-task learning model achieves better performances than the single-task models. This suggests that sharing information between two tasks is beneficial.

Effectiveness of concept selection. To study the effectiveness of involving concepts, we evaluate the performance of CAML-C. In CAML-C, we remove concept-level coattention pointer, set $\mathbf{e}_{u}$ to $\mathbf{d}_{u}^{\prime}$, set $\mathbf{e}_{v}$ to $\mathbf{d}_{v}^{\prime}$, and remove the concept relevant loss $\mathcal{L}_{c}$ from Eq. (18). From Tables 2 and 4 we can see that CAML-C consistently performs worse than or equal to CAML in terms of both explainability and accuracy. The results show the effectiveness of concept selection, especially for the explanation generation task.

\section{Conclusion}

In this paper, we propose a co-attentive multi-task learning model for explainable recommendation, which fully exploits the correlations between both recommendation and explanation tasks. We propose an encoder-selector-decoder architecture that is inspired by the cognitive process of human decision making. A hierarchical co-attentive selector is designed to effectively model the cross knowledge. Experiments show that our approach outperforms state-of-the-art baselines on both the prediction accuracy and generation quality. 


\section{References}

[Alvarez-Melis and Jaakkola, 2018] David Alvarez-Melis and Tommi S. Jaakkola. Towards robust interpretability with self-explaining neural networks. In NIPS, pages 7786-7795, 2018.

[Chen et al., 2018] Chong Chen, Min Zhang, Yiqun Liu, and Shaoping Ma. Neural attentional rating regression with review-level explanations. In $W W W$, pages 1583-1592, 2018.

[Chung et al., 2014] Junyoung Chung, Caglar Gulcehre, KyungHyun Cho, and Yoshua Bengio. Empirical evaluation of gated recurrent neural networks on sequence modeling. arXiv preprint arXiv:1412.3555, 2014.

[Erkan and Radev, 2004] Günes Erkan and Dragomir R Radev. Lexrank: Graph-based lexical centrality as salience in text summarization. Journal of artificial intelligence research, 22:457-479, 2004.

[He et al., 2015] Xiangnan He, Tao Chen, Min-Yen Kan, and Xiao Chen. Trirank: Review-aware explainable recommendation by modeling aspects. In CIKM, pages 16611670, 2015.

[Jang et al., 2017] Eric Jang, Shixiang Gu, and Ben Poole. Categorical reparameterization with gumbel-softmax. In ICLR, 2017.

[Kingma and Ba, 2015] Diederik P. Kingma and Jimmy Ba. Adam: A method for stochastic optimization. In ICLR, 2015.

[Koren, 2008] Yehuda Koren. Factorization meets the neighborhood: a multifaceted collaborative filtering model. In SIGKDD, pages 426-434, 2008.

[Lang, 2000] Annie Lang. The limited capacity model of mediated message processing. Journal of communication, 50(1):46-70, 2000.

[Lee and Seung, 2001] Daniel D Lee and H Sebastian Seung. Algorithms for non-negative matrix factorization. In NIPS, pages 556-562, 2001.

[Li et al., 2016] Jiwei Li, Michel Galley, Chris Brockett, Jianfeng Gao, and Bill Dolan. A diversity-promoting objective function for neural conversation models. In NAACL HLT, pages 110-119, 2016.

[Li et al., 2017] Piji Li, Zihao Wang, Zhaochun Ren, Lidong Bing, and Wai Lam. Neural rating regression with abstractive tips generation for recommendation. In SIGIR, pages 345-354, 2017.

[Lin, 2004] Chin-Yew Lin. Rouge: A package for automatic evaluation of summaries. Text Summarization Branches Out, 2004.

[McAuley and Leskovec, 2013] Julian McAuley and Jure Leskovec. Hidden factors and hidden topics: understanding rating dimensions with review text. In Proceedings of ACM conference on Recommender systems, pages 165172, 2013.
[Mnih and Salakhutdinov, 2008] Andriy Mnih and Ruslan R Salakhutdinov. Probabilistic matrix factorization. In NIPS, pages 1257-1264, 2008.

[Papineni et al., 2002] Kishore Papineni, Salim Roukos, Todd Ward, and Wei-Jing Zhu. Bleu: a method for automatic evaluation of machine translation. In $A C L$, pages 311-318, 2002.

[Peake and Wang, 2018] Georgina Peake and Jun Wang. Explanation mining: Post hoc interpretability of latent factor models for recommendation systems. In SIGKDD, pages 2060-2069, 2018.

[Rago et al., 2018] Antonio Rago, Oana Cocarascu, and Francesca Toni. Argumentation-based recommendations: Fantastic explanations and how to find them. In IJCAI, pages 1949-1955, 2018.

[Rendle, 2010] Steffen Rendle. Factorization machines. In ICDM, pages 995-1000, 2010.

[Sharma and Cosley, 2013] Amit Sharma and Dan Cosley. Do social explanations work?: Studying and modeling the effects of social explanations in recommender systems. In $W W W$, pages 1133-1144, 2013.

[Tay et al., 2018] Yi Tay, Luu Anh Tuan, and Siu Cheung Hui. Multi-pointer co-attention networks for recommendation. SIGKDD, 2018.

[Vig et al., 2009] Jesse Vig, Shilad Sen, and John Riedl. Tagsplanations: Explaining recommendations using tags. In International Conference on Intelligent User Interfaces, pages 47-56, 2009.

[Vinyals et al., 2015] Oriol Vinyals, Meire Fortunato, and Navdeep Jaitly. Pointer networks. In NIPS, pages 26922700, 2015.

[Wang et al., 2015] Zhongyuan Wang, Haixun Wang, JiRong Wen, and Yanghua Xiao. An inference approach to basic level of categorization. In CIKM, pages 653-662, 2015.

[Wang et al., 2018a] Xiang Wang, Xiangnan He, Fuli Feng, Liqiang Nie, and Tat-Seng Chua. Tem: Tree-enhanced embedding model for explainable recommendation. In $W W W$, pages 1543-1552, 2018.

[Wang et al., 2018b] Xiting Wang, Yiru Chen, Jie Yang, $\mathrm{Le} \mathrm{Wu}$, Zhengtao $\mathrm{Wu}$, and Xing Xie. A reinforcement learning framework for explainable recommendation. In ICDM, 2018.

[Williams, 1992] Ronald J Williams. Simple statistical gradient-following algorithms for connectionist reinforcement learning. Machine learning, 8(3-4):229-256, 1992.

[Wu et al., 2012] Wentao Wu, Hongsong Li, Haixun Wang, and Kenny Q Zhu. Probase: A probabilistic taxonomy for text understanding. In SIGMOD, pages 481-492, 2012.

[Zhang et al., 2014] Yongfeng Zhang, Guokun Lai, Min Zhang, Yi Zhang, Yiqun Liu, and Shaoping Ma. Explicit factor models for explainable recommendation based on phrase-level sentiment analysis. In SIGIR, pages 83-92, 2014. 